\title{
Epicardial Fat-mediated Inflammation: a Major Player in Cardiovascular Diseases
}

\author{
István Benedek and Theodora Benedek
}

University of Medicine and Pharmacy, Tîrgu Mureș, Romania

\section{CORRESPONDENCE}

Theodora Benedek

Str. Gheorghe Marinescu nr. 38

540139 Tîrgu Mureș, Romania

Tel: +40 265215551

E-mail: theodora.benedek@gmail.com
Epicardial fat has been recognized in recent years as one of the most relevant biomarkers associated with cardiovascular risk. The role of epicardial adipose tissue (EAT) in the determinism of several major cardiovascular diseases (CVD) has been explored by many clinical trials. According to these studies, EAT has been linked with the risk of coronary artery disease, atrial fibrillation (AF), unstable angina, and acute myocardial infarction. ${ }^{1-5}$ Furthermore, it has been demonstrated that patients with increased volumes of EAT present worse outcomes in the post-infarction period, as shown by a significantly lower left ventricular ejection fraction (LVEF) or an augmented ventricular remodeling process. ${ }^{6}$

\section{LOCAL AND SYSTEMIC PRO-INFLAMMATORY ACTIVITY OF THE EPICARDIAL FAT}

The mechanism by which EAT can play such a significant role in various CVDs has been attributed to the recently discovered property of this tissue to release pro-inflammatory cytokines in the systemic circulation. Such pro-inflammatory mediators include interleukines, gamma interferon, or alfa tumor necrosis factor. ${ }^{7,8}$ Also, EAT volume was demonstrated to be significantly larger in patients with increased serum levels of highly-sensitive C-reactive protein (hs-CRP), a biomarker expressing an increased systemic inflammation, proving the role of EAT as a mediator of systemic inflammation and demonstrating that EAT is a metabolically active tissue, releasing inflammatory mediators, which act not only on local but also on systemic level. 6,9

However, epicardial pericoronary fat was also associated in several studies with an increased systemic release of inflammatory biomarkers. Recent studies demonstrated that local EAT surrounding the coronary arteries can serve as a more reliable biomarker for coronary plaque vulnerability at that level. ${ }^{10}$ At the same time, it has been proved that the volume of the local EAT located in the vicinity of the left atrium can be associated with the risk for AF and with the risk of AF recurrence following a successful conversion to sinus rhythm. ${ }^{11-13}$ Furthermore, EAT secretes different adipokines and matrix metalloproteases 
with a pronounced fibrotic effect, stimulating the development of atrial fibrosis and structural changes in the atrial myocardium in the context of a complex atrial remodeling process, ultimately resulting in development of AF. ${ }^{13}$

\section{EAT-MEDIATED INFLAMMATION AS A MAJOR PLAYER IN CVD}

Two articles published in this number of JIM address the hypothesis of EAT-mediated inflammation as a major player in the determinism of CVDs. In an original article published by Cernea et al., the authors demonstrated that in diabetic patients, EAT was significantly higher in the group with decreased renal function or with reduced ejection fraction. According to the authors, EAT was significantly higher in the group with LVEF $<55 \%$ as compared to the group with LVEF $>55 \%(9.5 \pm 1.9 \mathrm{~mm}$ vs. $7.3 \pm 2.3$ $\mathrm{mm}$ ), at the same time being negatively correlated with eGFR ( $r=-0.293,95 \%$ CI: -0.531 to $0.013, p=0.33$ ). This study indicates that an increased EAT can serve as a reliable risk marker for renal injury and for deterioration of ventricular function in diabetic patients, suggesting that EAT-mediated inflammation could act on a systemic level, influencing not only the ventricular function but also the renal one. ${ }^{14}$

Another article published in this issue by Rat et al. presents a clinical update on the role of EAT in left atrial remodeling and genesis of AF, showing that EAT is associated not only with a higher risk for AF but also with a higher recurrence rate after successful catheter ablation of AF. ${ }^{15}$ This interesting new hypothesis indicates a potential role of EAT in the development of atrial remodeling, similar to previous observations on the correlation between EAT and left ventricular remodeling following an acute myocardial infarction. ${ }^{6}$ It is well-known that in the period following an acute myocardial infarction, an exacerbated inflammation is responsible for worse outcomes and ventricular remodeling. ${ }^{6,16,17}$ To what extent is this the result of the local release of inflammatory mediators from the area surrounding the infarcted tissue, as well as the link between local activity of the epicardial fat and atrial remodeling in AF, remains to be established.

\section{EPICARDIAL FAT AND CORONARY PLAQUE VULNERABILITY}

While the role of EAT in the inflammation-mediated progression of coronary artery disease has been recognized, its potential role in the vulnerabilization of unstable coronary plaques is still under debate, similarly to the role of locally accumulated EAT in functional destabilization of neighboring structures, such as the atria (in which case it can trigger a new onset of AF or its recurrence) or the coronary arteries (in which case it can trigger an acute coronary event). ${ }^{18,19}$ An interesting approach for the study of EAT and its role in inflammation-mediated reactions is represented by the identification of specific microRNAs associated with plaque vulnerability from the samples of epicardial fat tissue collected during open heart surgery. Preliminary data suggest that such microRNAs are present in a higher amount in the close vicinity of unstable coronary plaques, indicating a potential role of locally released inflammatory mediators originating from the epicardial fat in the determinism of acute coronary syndromes. ${ }^{20,21}$

\section{IMAGING TOOLS FOR QUANTIFICATION OF METABOLICALLY ACTIVE FAT TISSUES}

In an attempt to identify the best imagistic method for quantification of EAT, various modern methods have been proposed and studied. Computed cardiac angiography demonstrated its superiority over cardiac magnetic resonance and traditional echocardiography, being able to quantify with high accuracy global EAT volumes and local volumes of fat surrounding different structures, such as the left and right atrium or the coronary arteries. $5,22-24$

But epicardial fat is not the only metabolically active fat involved in the development of inflammatory reactions. Pericardial fat, as well as the fat accumulated in different regions of the body, could also be easily quantified by CT and can express an increased risk for different cardiovascular events. Alternative methods for fat quantification have also been developed, relying on complex methods for post-processing cardiac CT data. Such alternatives include quantification of thoracic fat, abdominal fat, or pericardial fat. ${ }^{25}$ However, none of them demonstrated so far a clear superiority of these approaches over epicardial fat in predicting cardiovascular risk.

This issue of JIM expresses the great interest showed recently towards epicardial fat, an imaging-derived biomarker that can serve as a reliable imagistic tool for assessing cardiovascular risk. Current studies show promising results in evaluating the role of this new biomarker to predict future cardiac events, and the two articles published on this actual topic in this issue of the journal reflect the great expectations from this new imagingderived powerful tool. However, further studies are required to validate the role of EAT as a routine biomarker in clinical settings. 


\section{REFERENCES}

1. Talman AH, Psaltis PJ, Cameron JD, Meredith IT, Seneviratne SK, Wong DTL. Epicardial adipose tissue: far more than a fat depot. Cardiovasc Diagn Ther. 2014;4:416-429.

2. Sinha SK, Thakur R, Jha MJ, et al. Epicardial Adipose Tissue Thickness and Its Association With the Presence and Severity of Coronary Artery Disease in Clinical Setting: A Cross-Sectional Observational Study. J Clin Med Res. 2016:8:410-419.

3. Picard FA, Gueret P, Laissy JP, et al. Epicardial adipose tissue thickness correlates with the presence and severity of angiographic coronary artery disease in stable patients with chest pain. PLoS One. 2014;9:e110005.

4. Gaeta M, Bandera F, Tassinari F, et al. Is epicardial fat depot associated with atrial fibrillation? A systematic review and meta-analysis. Europace. 2017:19:747-752.

5. Benedek T, Opincariu D, Rat N, Hodas R, Mester A, Benedek I. The Assessment of Epicardial Adipose Tissue in Acute Coronary Syndrome Patients. A Systematic Review. Journal of Cardiovascular Emergencies. 2017;3:18-29.

6. Opincariu D, Mester A, Dobra M, et al. Prognostic value of epicardial fat thickness as a biomarker of increased inflammatory status in patients with type 2 diabetes mellitus and acute myocardial infarction. Journal of Cardiovascular Emergencies. 2015;1:11-18.

7. Sacks HS, Fain JN. Human epicardial fat: what is new and what is missing? Clin Exp Pharmacol Physiol. 2011;38:879-87.

8. lacobellis G, Bianco AC. Epicardial adipose tissue: emerging physiological, pathophysiological and clinical features. Trends Endocrinol Metab. 2011;22:450-457

9. Gitsioudis G, Schmahl C, Missiou A, et al. Epicardial Adipose Tissue Is Associated with Plaque Burden and Composition and Provides Incremental Value for the Prediction of Cardiac Outcome. A Clinical Cardiac Computed Tomography Angiography Study. PLOS ONE. 2016;11:e0155120.

10. Sen F, Yilmaz S, Balci K, et al. The Relationship Between Epicardial Adipose Tissue Thickness and Infarct-Related Artery Patency in Patients with ST-segment Elevation Myocardial Infarction. Angiology. 2016;67:281286.

11. Bos D, Vernooij MW, Shahzad R, et al. Epicardial Fat Volume and the Risk of Atrial Fibrillation in the General Population Free of Cardiovascular Disease. JACC Cardiovasc Imaging. 2017;10:1405-1407.

12. Batal $O$, Schoenhagen $P$, Shao M, et al, Left atrial epicardial adiposity and atrial fibrillation. Circ Arrhythm Electrophysiol. 2010;3:230-236.
13. Al Chekakie MO, Akar JG. Epicardial Fat and Atrial Fibrillation: A Review. J Atr Fibrillation. 2012;4:483.

14. Cernea S, Blendea C, Roiban AL, Benedek T. Cardio-renal Correlations and Epicardial Adipose Tissue in Patients with Type 2 Diabetes. Journal of Interdisciplinary Medicine. 2017;2:312-319.

15. Rat N, Mitre A, Korodi S, et al. Epicardial Fat, Paracrine-mediated Inflammation and Atrial Fibrillation. Journal of Interdisciplinary Medicine. 2017:2:304-307.

16. Tscharre M, Hauser C, Rohla M, et al. Epicardial adipose tissue and cardiovascular outcome in patients with acute coronary syndrome undergoing percutaneous coronary intervention. Eur Heart J Acute Cardiovasc Care. 2017;6:750-752

17. Tanindi A, Kocaman S, Erkan A, et al. Epicardial adipose tissue thickness is associated with myocardial infarction and impaired coronary perfusion. Anatol J Cardiol. 2015;15:224-231.

18. Nakanishi K, Fukuda S, Tanaka A, et al. Persistent epicardial adipose tissue accumulation is associated with coronary plaque vulnerability and future acute coronary syndrome in non-obese subjects with coronary artery disease. Atherosclerosis. 2014;237:353-360.

19. Park J, Choi S, Zheng M, et al. Epicardial adipose tissue thickness is a predictor for plaque vulnerability in patients with significant coronary artery disease. Atherosclerosis. 2013:226:134-139.

20. Liu Y, Fu W, Lu M, Huai S, Song Y, Wei Y. Role of miRNAs in Epicardial Adipose Tissue in CAD Patients with T2DM. BioMed Research International. 2016;2016:1629236.

21. Vacca M, Di Eusanio M, Cariello $N$, et al. Integrative miRNA and wholegenome analyses of epicardial adipose tissue in patients with coronary atherosclerosis. Cardiovasc Res. 2016;109:228-239.

22. Bertaso A, Bertol D, Duncan B, Foppa M. Epicardial Fat: Definition, Measurements, and Systematic Review of Main Outcomes. Arq Bras Cardiol. 2013;101:18-28.

23. lacobellis $G$, Willens $H$. Echocardiographic Epicardial Fat: A Review of Research and Clinical Applications. J Am Soc Echocardiogr. 2009;22:13111319.

24. Shmilovich $H$, Dey $D$, Cheng $\vee$, et al. Threshold for the upper normal limit of indexed epicardial fat volume: derivation in a healthy population and validation in an outcome based study. Am J Cardiol. 2011;108:1680-1685.

25. Rosito GA Massaro J, Hoffmann U et al. Pericardial fat, visceral abdomina fat, cardiovascular disease risk factors, and vascular calcification in a community-based sample: the Framingham Heart Study. Circulation. 2008;117:605-613. 